\title{
Measurement of thiopurine methyltransferase activity and azathioprine metabolites in patients with inflammatory bowel disease
}

\author{
P W Lowry, C L Franklin, A L Weaver, M Gennett Pike, D C Mays, W J Tremaine,
} J J Lipsky, W J Sandborn

\begin{abstract}
Background-Measurement of 6-thioguanine nucleotide concentrations may be useful for optimising treatment with azathioprine and 6-mercaptopurine.

Methods-We conducted a study of 170 patients with inflammatory bowel disease treated with azathioprine or 6-mercaptopurine to determine the relationship between 6-thioguanine nucleotide concentrations and both disease activity, as measured by the inflammatory bowel disease questionnaire (active disease $<170$, remission $\geqslant 170$ ) and leucopenia. Blood was submitted for whole blood 6-thioguanine nucleotide concentration and leucocyte count.

Results-Mean (SD) inflammatory bowel disease questionnaire score was 176 (32). There was no correlation between inflammatory bowel disease questionnaire scores and 6-thioguanine nucleotide concentrations $\left(r_{\mathrm{s}}=-0.09, \mathrm{p}=0.24\right)$. Median 6-thioguanine nucleotide concentrations in 56 patients with active disease and 114 patients in remission were similar (139 $v$ $131 \mathrm{pmol} / 8 \times 10^{8}$ red blood cells; $\left.\mathrm{p}=0.26\right)$. There was no correlation between 6-thioguanine nucleotide concentrations and leucocyte counts.

Conclusions-In patients with inflammatory bowel disease treated with azathioprine or 6-mercaptopurine, 6-thioguanine nucleotide concentrations did not correlate with disease activity, as measured by the inflammatory bowel disease questionnaire, or leucocyte count. These findings are discrepant with most previous studies, possibly due to selection of responding patients who tolerated the medications. A prospective, randomised, dose optimisation trial using 6-thioguanine nucleotide concentrations is warranted.

(Gut 2001;49:665-670)
\end{abstract}

Keywords: azathioprine; 6-mercaptopurine; thioguanine nucleotides; thiopurine methyltransferase; inflammatory bowel disease

Azathioprine (AZA) and 6-mercaptopurine (6-MP) have emerged as important therapies for both induction and maintenance of remission in patients with Crohn's disease (CD) and ulcerative colitis (UC). ${ }^{12}$ Azathioprine is rapidly converted to 6-MP after absorption, and 6-MP is subsequently metabolised to compounds with immune modifier activity, the 6-thioguanine nucleotides (6-TGN), as well as 6-methylmercaptopurine (6-MMP) which is thought to be inactive. ${ }^{3}$ One major catabolic enzyme of AZA and 6-MP is thiopurine methyltransferase (TPMT). The activity of TPMT appears to have a trimodal distribution in the general population with low activity $(<5.0$ $\mathrm{U} / \mathrm{ml}$ red blood cells (RBCs)) occurring at a frequency of $0.3 \%$; intermediate activity (5.013.7 U/ml RBCs) occurring at a frequency of $11.1 \%$; and normal or high activity (13.8-25.1 $\mathrm{U} / \mathrm{ml} \mathrm{RBCs}$ ) occurring at a frequency of $88.6 \%{ }^{4}$ Among patients with acute lymphoblastic leukaemia (ALL), low baseline levels of TPMT activity led to a shift in metabolism of 6-MP away from production of the inactive metabolite 6-MMP and towards production of the "active" metabolite 6-TGN.

The therapeutic mechanism of action of 6-TGN is unclear; it may modify the immune system by incorporation into DNA, resulting in breaks in strands of DNA. ${ }^{3}$ The primary toxicity associated with AZA and 6-MP is leucopenia, possibly due to accumulation of 6-TGN in neutrophils. ${ }^{6}$ In patients with ALL there is a positive correlation between RBC 6-TGN concentration and risk of leucopenia. ${ }^{7}$ This association has been confirmed in some $\mathrm{e}^{8-10}$ but not all ${ }^{11}$ studies of patients with UC and CD. Patients with ALL who have a RBC 6-TGN concentration that falls above the median value of $275 \mathrm{pmol} / 8 \times 10^{8} \mathrm{RBCs}$ have a significantly greater rate of survival. ${ }^{12}$ Similarly, some ${ }^{8}{ }^{9}$ but not all ${ }^{11}$ studies of patients with UC or CD have demonstrated an association between higher RBC 6-TGN concentrations and improvement in disease activity. We present the results of the largest study to date in patients with inflammatory bowel disease (IBD) which examines the relationship between pretreatment TPMT activity, AZA and 6-MP dose, whole blood concentrations of AZA and 6-MP metabolites, and disease activity and toxicity (including risk of leucopenia).

\section{Methods}

PATIENT SELECTION

Potential patients were identified by cross referencing a Mayo Clinical Laboratory

Abbreviations used in this paper: 6-TGN 6-thioguanine nucleotides; AZA, azathioprine; 6-MP, 6-mercaptopurine; CD, Crohn's disease; UC, ulcerative colitis; 6-MMP, 6-methylmercaptopurine; TPMT, thiopurine methyltransferase; RBC, red blood cell; ALL, acute lymphoblastic leukaemia; IBD, inflammatory bowel disease; IBDQ, inflammatory bowel disease questionnaire; WBC, white blood cell; 5-ASA, 5-aminosalicylic acid. 
database of all patients who had RBC TPMT activity measured between 1992 and June 1998 with an institutional database of all patients with IBD evaluated at Mayo Clinic Rochester (identified by ICD9 code diagnoses for CD and UC) during that same time period. The medical records of these patients were reviewed for eligibility criteria. Eligible patients were outpatients of either sex, aged 18 years or older, with an established diagnosis of CD or UC, who underwent an evaluation at Mayo Clinic Rochester between 1992 and June 1998. In addition, eligible patients either started or continued on treatment with AZA or 6-MP at the time of their last Mayo Clinic visit (irrespective of the duration of therapy), were currently taking AZA or 6-MP at the time of study enrolment (see below), had a baseline RBC TPMT activity determination prior to starting treatment with AZA or 6-MP, agreed to comply with the study protocol, and gave written informed consent. An introductory letter explaining the study was sent, and patients who were currently taking AZA or 6-MP and who agreed to participate were sent additional study materials. The study was approved by the institutional review board of the Mayo Clinic.

\section{STUDY DESIGN}

Subjects were asked to complete a self administered survey to obtain the following information: sex; age; weight; all IBD medications (including doses and month and year when medications were started); and all past dose adjustments of AZA or 6-MP (and the reason for each AZA or 6-MP dose adjustment). Subjects also completed a self administered quality of life assessment (inflammatory bowel disease questionnaire (IBDQ)) consisting of four main categories (bowel function, emotional status, systemic symptoms, and social function). This questionnaire has been previously validated, and the total score ranges from 32 to 224, with higher scores indicating better quality of life. Patients in clinical remission have minimal scores of $170 .{ }^{13}$ The patients' medical records were abstracted to obtain the following information: demographic data; type of IBD and date of diagnosis; pretreatment RBC TPMT enzyme activity; disease location; past surgical resections; start date and initial dose of AZA or 6-MP; dates of all subsequent dose adjustments of AZA or 6-MP, and reasons for dose adjustments; lowest recorded white blood cell (WBC) count for each dose of AZA or 6-MP; and other concurrent IBD medications. Subjects mailed in a sample of whole blood (overnight delivery) along with their completed survey. They were instructed to undergo phlebotomy one hour after their usual daily dose of AZA or 6-MP; if patients were receiving sulphasalazine, mesalamine, or olsalazine, it was recommended that they administer a dose one hour before blood withdrawal.

The following measurements were made on blood: complete blood count with leucocyte differential; RBC TPMT enzyme activity; whole blood 6-TGN and 6-MMP concentrations; and for selected patients receiving sulphasalazine, mesalamine, or olsalazine, measurement of plasma sulphasalazine, 5-aminosalicylic acid (5-ASA, mesalamine), and $\mathrm{N}$-acetyl-5-ASA concentrations, as appropriate. RBC TPMT activity was determined by a radioimmunoassay method, as previously described (Mayo Medical Laboratories, Rochester, MN, USA). ${ }^{14}$ Whole blood total 6-TGN was assayed by high pressure liquid chromatography using the Erdmann method with minor modifications. ${ }^{15}$ Whole blood rather than washed RBCs was used, following a study that determined that total 6-TGN concentration is not significantly different in whole blood compared with washed RBCs (unpublished data). Whole blood total 6-TGN concentration represents the total 6-TGN content, consisting of a composite of mono-, di-, and triphosphate nucleotides. Whole blood 6-TGN concentration is normalised to $8 \times 10^{8} \mathrm{RBCs}$. Plasma concentrations of sulphasalazine, 5-ASA, and $\mathrm{N}$-acetyl5-ASA were determined by methods previously described, with minor modifications. ${ }^{16-18}$

\section{STATISTICAL ANALYSIS}

Standard statistical methods were used to evaluate various univariate relationships. Correlations were assessed based on the Spearman rank correlation coefficient and group differences were evaluated based on the two sample $t$ test, Wilcoxon rank sum test, or the $\chi^{2}$ test, as appropriate. All calculated $\mathrm{p}$ values were two sided and $p$ values $<0.05$ were considered statistically significant.

\section{Results}

A total of 951 patients with IBD had TPMT activity measured between 1992 and June 1998. These patients' medical records were reviewed during the fall of 1998 for eligibility criteria. We identified 376 patients who had either started or continued AZA or 6-MP at the time of their last Mayo Clinic evaluation. These 376 patients were contacted by mail regarding the study, of whom 217 initially agreed to participate and 170 actually returned the questionnaires and submitted blood specimens; 575 patients were ineligible. Detailed records of the reasons for ineligibility were not kept but reasons included: patient not started on AZA or 6-MP; patient no longer taking AZA or 6-MP because of failure to respond; AZA or 6-MP discontinued after operation; patient discontinued AZA or 6-MP because of toxicity; and other miscellaneous reasons.

Table 1 Patient characteristics

\begin{tabular}{ll}
\hline Characteristic & \\
\hline Sex (M/F) & $80 / 90$ \\
Age (y) (mean (range)) & $42.9(18-81)$ \\
Type of disease & 130 \\
$\quad$ Crohn's disease & 40 \\
$\quad$ Ulcerative colitis & $12.2(0.6-50.5)$ \\
Duration of disease (y) (mean (range)) & 78 \\
Previous intestinal resection (n) & 35 \\
Disease site (n) & 46 \\
$\quad$ Ileum & $89(\mathrm{CD} 49, \mathrm{UC} 40)$ \\
$\quad$ Ileocolon & $176(75-223)$ \\
$\quad$ Inflammatory bowel disease & \\
$\quad$ questionnaire (mean (range)) & \\
\hline
\end{tabular}


Table 2 Azathioprine/6-mercaptopurine doses

\begin{tabular}{lll}
\hline Drug & Dose issues & Result \\
\hline Azathioprine & No patients & 115 \\
& Starting dose (mg/kg/day) & \\
& Mean & 1.9 \\
& Range & $0.5-4.0$ \\
& Current dose (mg/kg/day) & \\
& $\quad$ Mean & 1.9 \\
& Range & $0.5-4.1$ \\
& Duration (months) & \\
$\quad$ Mean & 21.7 \\
& Range & $3.5-102.1$ \\
6-Mercaptopurine & No patients & 55 \\
& Starting dose (mg/kg/day) & \\
$\quad$ Mean & 0.9 \\
$\quad$ Range & $0.3-1.9$ \\
& Current dose (mg/kg/day) & 1.1 \\
$\quad$ Mean & $0.3-3.3$ \\
& Range & \\
& Duration (months) & 41.4 \\
$\quad$ Mean & $3.7-81.2$ \\
\hline & Range &
\end{tabular}

PATIENT CHARACTERISTICS

The characteristics of the study participants are described in table 1. Eighty (47\%) were male. At the time of the study, mean age was 42.9 years (range 18-81). A total of $130(77 \%)$ patients had CD and 40 (24\%) had UC. Mean (SD) duration of disease was 12.2 (9.7) years (range $0.6-50.5)$. Seventy eight (46\%) patients had undergone a previous ileal and/or colonic resection.

TREATMENT WITH AZATHIOPRINE AND

6-MERCAPTOPURINE

At the time of study participation, 115 (68\%) patients were taking AZA and 55 (32\%) were taking 6-MP, as shown in table 2. A total of 108 patients were started on AZA and three were subsequently changed to $6-\mathrm{MP} ; 62$ patients were started on 6-MP and 10 were later switched to AZA. Mean (SD) starting AZA and 6-MP doses were $1.9(0.6)$ and $0.9(0.3)$ $\mathrm{mg} / \mathrm{kg} /$ day, respectively (table 2 ). There was no significant correlation between the initial thiopurine dose and pretreatment TPMT activity (115 patients started on AZA, $r_{\mathrm{s}}=0.01, \mathrm{p}=0.90$; 55 patients started on 6-MP, $r_{\mathrm{s}}=-0.09$, $\mathrm{p}=0.55)$. Furthermore, the initial 6-MP dose was similar in patients with heterozygous intermediate $(>13.7 \mathrm{U} / \mathrm{ml})$ and homozygous normal (5.1-13.7 U/ml) TPMT activity (mean (SD) $0.90 \quad(0.3) \quad v \quad 0.89 \quad(0.2) \quad \mathrm{mg} / \mathrm{kg} /$ day, respectively). Likewise, the initial AZA dose was similar between patients with heterozygous intermediate and homozygous normal TPMT activity (1.89 (0.5) v $1.84(0.9) \mathrm{mg} / \mathrm{kg} / \mathrm{day}$, respectively). At the time of their study participation, mean AZA and 6-MP doses were 1.9 (0.6) and $1.1(0.5) \mathrm{mg} / \mathrm{kg} /$ day, respectively.
Mean duration of AZA use was 21.7 (16.6) months (range 3.5-102.1), and mean duration of 6-MP use was 41.4 (19.6) months (range 3.7-81.2) (table 2). A total of 33 patients $(19 \%)$ had their AZA or 6-MP dose adjusted or temporarily discontinued on at least one occasion due to a decrease in WBC count; seven patients $(4 \%)$ required two dose changes and three patients $(2 \%)$ required three or more dose changes. The mean total WBC count at the time of a dose change was $3.1 \times 10^{6} / 1$ (range 1.5-4.3). One patient required a dose change due to thrombocytopenia. Other side effects requiring AZA or 6-MP dose adjustment included liver test abnormalities in eight patients $(5 \%)$, nausea or vomiting in seven patients $(4 \%)$, pancreatitis in one patient $(1 \%)$, and rash in one patient $(1 \%)$.

THIOPURINE METHYLTRANSFERASE ACTIVITY

TPMT activity measured at the time of the study after blood withdrawal (for 164/170 patients) was distributed as follows: 141 patients $(83 \%)$ were in the homozygous high (normal) range $(>13.7 \mathrm{U} / \mathrm{ml}), 23$ patients $(14 \%)$ were in the heterozygous intermediate range $(5.1-13.7 \mathrm{U} / \mathrm{ml})$, and no patient was in the homozygous low range $(\leqslant 5.1 \mathrm{U} / \mathrm{ml})$. Mean pretreatment TPMT activity was higher than the current (study) TPMT activity (18.5 (4.5) v 17.3 (3.8) $\mathrm{U} / \mathrm{ml} ; \mathrm{p}<0.001$, paired $t$ test). Mean interval between pretreatment and current study TPMT activity measurements was 2.2 years (range 0.3-6.6). Additional details regarding TPMT activity are shown in table 3.

\section{AZATHIOPRINE AND 6-MERCAPTOPURINE} METABOLITES

Among all patients participating in the study, median whole blood 6-TGN concentration was $136 \mathrm{pmol} / 8 \times 10^{8} \mathrm{RBCs}$ (mean (SD) 163 (99), range 12-564) and median whole blood 6-MMP concentration was $771 \mathrm{pmol} / 8 \times 10^{8}$ RBCs (mean (SD) 2190 (3644), range 58-23 294) (table 4). Patients with TPMT activity in the intermediate range (heterozygous for low and high activity alleles) had significantly greater whole blood 6-TGN concentrations than patients with TPMT activity in the normal range (homozygous for high activity alleles): mean (SD) whole blood 6-TGN concentrations for each group were 253 (136) and 151 (84) pmol $/ 8 \times 10^{8} \mathrm{RBCs}$, respectively $(\mathrm{p}=0.002)$ (table 4$)$. There was a trend towards

Table 3 Thiopurine methyltransferase (TPMT) activy

\begin{tabular}{|c|c|c|c|c|c|c|}
\hline & \multicolumn{3}{|c|}{ Pretreatment TPMT activity (U/ml) } & \multicolumn{3}{|c|}{ Current (study) TPMT activity (U/ml) } \\
\hline & Overall & $\begin{array}{l}\text { Homozygous } \\
\text { normal (>13.7) }\end{array}$ & $\begin{array}{l}\text { Heterozygous } \\
\text { intermediate } \\
(5.1-13.7)\end{array}$ & Overall & $\begin{array}{l}\text { Homozygous } \\
\text { normal (>13.7) }\end{array}$ & $\begin{array}{l}\text { Heterozygous } \\
\text { intermediate } \\
(5.1-13.7)\end{array}$ \\
\hline No & 163 & 145 & 18 & 164 & 141 & 23 \\
\hline Mean (SD) & $18.5(4.5)$ & $19.6(3.5)$ & $10.0(1.7)$ & $17.3(3.8)$ & $18.4(2.7)$ & $10.5(1.7)$ \\
\hline Median & 18.7 & 19.2 & 9.8 & 17.4 & 18.0 & 9.9 \\
\hline IQR & $16.3,21.0$ & $17.5,21.4$ & $9.1,11.2$ & $15.7,20.1$ & $16.3,20.1$ & $9.2,12.0$ \\
\hline Range & $6.8,39.1$ & $13.9,39.1$ & $6.8,12.9$ & $7.9,28.9$ & $13.9,28.9$ & $7.9,13.7$ \\
\hline
\end{tabular}

IQR, interquartile range, 25 th and 75 th percentiles. 
Table 4 Red blood cell (RBC) 6-thioguanine nucleotide (6-TGN) concentrations

\begin{tabular}{|c|c|c|}
\hline Patient group & No patients & 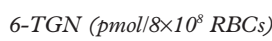 \\
\hline All patients & 170 & $\begin{array}{l}\text { Median } 136 \\
\text { Mean (SD) } 163 \text { (99) } \\
\text { Range 12-564 }\end{array}$ \\
\hline Intermediate range TPMT activity* & 23 & $\begin{array}{l}\text { Median } 282 \\
\text { Mean (SD) } 253 \text { (136) }\end{array}$ \\
\hline High range TPMT activity* & 141 & $\begin{array}{l}\text { Median } 130 \\
\text { Mean (SD) } 151(84)^{\star \star}\end{array}$ \\
\hline Patients with active disease (IBDQ $<170$ ) & 56 & $\begin{array}{l}\text { Median } 139 \\
\text { Mean (SD) } 178 \text { (116) }\end{array}$ \\
\hline Patients in clinical remission (IBDQ $\geqslant 170$ ) & 114 & $\begin{array}{l}\text { Median } 131 \\
\text { Mean (SD) } 156 \text { (89) }\end{array}$ \\
\hline
\end{tabular}

${ }^{\star}$ Six patients did not undergo TPMT testing.

${ }^{\star} \star$ Patients with intermediate TPMT activity levels were more likely to have high whole blood 6-TGN concentrations $(\mathrm{p}=0.002)$.

IBDQ, inflammatory bowel disease questionnaire.

a greater mean whole blood 6-TGN concentration in patients receiving concurrent mesalamine $(n=58)$, sulphasalazine $(n=2)$, or olsalazine $(n=1)$ compared with patients not receiving these medications $(182$ (110) $v 153$ (91) $\mathrm{pmol} / 8 \times 10^{8} \mathrm{RBCs} ; \mathrm{p}=0.10$ ). There was no correlation between whole blood 6-TGN concentration and whole blood 6-MMP concentration $\left(r_{\mathrm{s}}=0.04, \mathrm{NS}\right)$.

RISK FACTORS FOR LEUCOPENIA

Among the 168 patients in whom a WBC value from the time of the study was available, the mean (SD) WBC count was $5.4(1.8) \times 10^{9} / 1$ (range 2.4-12.6). Thirty four patients (20\%) had a WBC count $<4.0 \times 10^{9} / 1$ and six patients $(4 \%)$ had a WBC count $<3.0 \times 10^{9} / 1$. Patients receiving mesalamine, sulphasalazine, or olsalazine concurrently had a significantly lower (but still normal) median WBC count compared with patients not taking these medications $\left(4.5 \times 10^{9} / 1 \vee 5.4 \times 10^{9} / 1 ; \mathrm{p}=0.02\right)$. The following factors were not significantly associated with WBC or absolute neutrophil count at the time blood was obtained for the study: pretreatment or current TPMT activity; AZA or 6-MP dose $(\mathrm{mg} / \mathrm{kg})$; or whole blood 6-TGN concentration $(p>0.05)$. Baseline RBC TPMT activity was not significantly associated with subsequent occurrence of leucopenia (WBC $<4.0 \times 10^{9} / 1$ or WBC $\left.<3.0 \times 10^{9} / 1\right)$.

ACTIVITY OF INFLAMMATORY BOWEL DISEASE Among all study participants, mean (SD) IBDQ score was 176 (32) (range 75-223) (table 1). There was no significant correlation between disease activity (IBDQ point score) and whole blood 6-TGN concentration $\left(r_{\mathrm{s}}=-0.09, \mathrm{p}=0.24\right)$, as shown in fig 1. Comparing 56 patients with more active disease (IBDQ $<170$ ) versus 114 patients in clinical remission (IBDQ $\geqslant 170$ ), median whole blood 6-TGN concentrations were similar (139 $v 131 \mathrm{pmol} /$ $8 \times 10^{8}$ RBCs, respectively; $\mathrm{p}=0.26$ ) (table 4). Similarly, there was no correlation between disease activity and current (study) TPMT activity level $\left(r_{\mathrm{s}}=-0.05, \mathrm{p}=0.55\right)$. Mean $(\mathrm{SD})$ current TPMT activity was 17.3 (3.6) $\mathrm{U} / \mathrm{ml}$ among the 56 patients with active disease compared with 17.3 (3.9) $\mathrm{U} / \mathrm{ml}$ among the 114 patients in clinical remission. Furthermore, $33 \%$ of patients with TPMT activity in the homozygous normal range had active disease compared with $39 \%$ of patients with TPMT

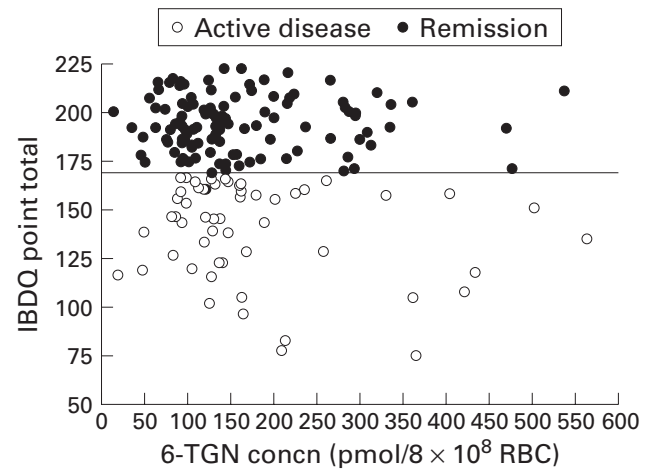

Figure 1 Relationship between inflammatory bowel disease questionnaire (IBDQ) score and red blood cell (RBC) 6-thioguanine nucleotide (6-TGN) concentration in patients with active disease and in those in remission.

activity in the heterozygous intermediate range. There was also no significant correlation between IBDQ score and RBC 6-MMP concentration, AZA or 6-MP dose $(\mathrm{mg} / \mathrm{kg} /$ day), or WBC or neutrophil count at the time blood was obtained for the study.

PLASMA 5-AMINOSALICYLIC ACID AND N-ACETYL5-AMINOSALICYLIC ACID CONCENTRATIONS Among the 61 patients receiving mesalamine, sulphasalazine, or olsalazine, 56 had plasma 5-ASA and $\mathrm{N}$-acetyl-5-ASA concentrations determined; four of these patients had concentrations undetectable or too low to accurately quantify. For the remaining 52 patients, median plasma 5-ASA concentration was 5.7 $\mathrm{mol} / \mathrm{l}$ (range 0.14-85.7), and median plasma $\mathrm{N}$-acetyl-5-ASA concentration was $9.8 \mathrm{~mol} / \mathrm{l}$ (range 0.2-71.0).

\section{Discussion}

In patients with ALL receiving 6-MP chemotherapy, the active metabolite 6-TGN is considered to exert its therapeutic effect through incorporation into DNA, with subsequent disruption of cell replication. Routine measurement of RBC 6-TGN concentration has been advocated as a means of assessing 6-MP drug effect, and paediatric patients with ALL receiving 6-MP at a dose of $75 \mathrm{mg} / \mathrm{m}^{2}$ are more likely to relapse if RBC 6-TGN concentration falls below the median value of 275 pmol $/ 8 \times 10^{8}$ RBCs. ${ }^{12}$ Likewise, this patient group is more likely to experience leucopenia if RBC 6-TGN concentrations are elevated. ${ }^{7}$

Our study suggests that some caution should be used in extrapolating these findings to patients with IBD receiving immune modifier therapy with AZA or 6-MP. One recent study of paediatric patients with IBD treated with 6-MP reported that the median RBC 6-TGN concentration in patients with clinical response was $312 \mathrm{pmol} / 8 \times 10^{8} \mathrm{RBCs}$ whereas the median RBC 6-TGN concentration in non-responders was $199 \mathrm{pmol} / 8 \times 10^{8} \mathrm{RBCs}^{9}{ }^{9}$ These authors suggested a target RBC 6-TGN concentration exceeding $230 \mathrm{pmol} / 8 \times 10^{8} \mathrm{RBCs}$ to optimise induction and maintenance of remission. In contrast, in our study we found no correlation between whole blood 6-TGN concentrations and remission and no difference in median 
whole blood 6-TGN concentrations in patients in clinical remission compared with those with active disease (131 $\left.v 139 \mathrm{pmol} / 8 \times 10^{8} \mathrm{RBCs}\right)$. While the median whole blood 6-TGN concentration of $136 \mathrm{pmol} / 8 \times 10^{8} \mathrm{RBCs}$ in our study was lower than the RBC 6-TGN concentrations reported in previous studies which used a different assay (see below), ${ }^{89}$ the majority of the patients in our study $(67 \%)$ were in clinical remission, suggesting that the AZA and 6-MP doses used, and whole blood 6-TGN concentrations achieved, were sufficient to achieve the desired clinical effect in most patients. In addition, there was no correlation between whole blood 6-TGN concentrations and total WBCs. Our data are similar to those of another recent study that assessed the efficacy of an intravenous loading dose of AZA compared with standard oral dosing. ${ }^{11}$ In this latter study, in which mean steady state RBC 6-TGN concentrations for the intravenous loading dose and oral AZA groups were 164 and $124 \mathrm{pmol} / 8 \times 10^{8} \mathrm{RBCs}$, respectively, there was no correlation between RBC 6-TGN concentrations and either clinical response or occurrence of leucopenia.

There are a variety of potential explanations for these discrepant results. Previous studies were smaller, ranging in size from 25 to 96 patients. $^{8911}$ Two of the previous studies evaluated only paediatric patients ${ }^{89}$ whereas another study $^{11}$ and our current study included only adults. In the current study, disease activity was measured by the IBDQ score whereas in previous studies disease activity was measured by the Harvey-Bradshaw index ${ }^{89}$ and the CD activity index. ${ }^{11}$ In the two studies that measured disease activity with the HarveyBradshaw index, different definitions of remission were used (score $<4$ and score $<5$ ) ${ }^{8}{ }^{9}$ The correlations between IBDQ and both the Harvey Bradshaw index and $C D$ activity index are only -0.55 and -0.67 , respectively, ${ }^{13}$ although all three have been well validated as measures of disease activity. In one of the previous studies, patients were studied for the first four months of AZA therapy. ${ }^{11}$ In two other studies patients were treated with $6-\mathrm{MP}$ for a minimum of four months (thus patients with early dose dependent toxicity from AZA or 6-MP were not included). In the current study, patients currently receiving AZA or 6-MP for any period of time were offered enrolment but no patients treated for less than 3.5 months participated. The majority of patients in the current study and two of the previous studies ${ }^{89}$ were in symptomatic remission on maintenance therapy with AZA or 6-MP, in comparison with patients in another previous study who were starting AZA for active disease. ${ }^{11}$ Patients with low or intermediate TPMT activity were excluded from one previous study. ${ }^{11}$ Physicians caring for patients in the current study may have elected to use lower initial doses of AZA or 6-MP in patients with intermediate or low normal TPMT activity (this is nearly impossible to determine after the fact because the decision may not be recorded in the medical record). Multiple sampling of patients in one of the studies may have led to an overestimate of the beneficial effect of RBC 6-TGN monitoring. ${ }^{9}$ These differences in study design, with differing results, make it difficult to determine precisely the role of 6-TGN monitoring at the present time in patients with IBD treated with AZA or 6-MP. The negative outcome of our study suggests that additional research is needed before this strategy for optimising therapy for patients with IBD is adopted into routine clinical practice.

Studies of 6-TGN therapeutic drug monitoring in patients with IBD which reported an association between elevated 6-TGN concentrations and both efficacy and leucopenia used an assay for RBC 6-TGN developed by Lennard and Maddocks. ${ }^{19}$ We used a different RBC 6-TGN assay developed by Erdmann and colleagues ${ }^{15}$ and adapted it to whole blood, and thus our results are not directly comparable. However, we demonstrated a high degree of correlation in a blinded comparison of the two assays, with a conversion factor of approximately 1.6 to convert Erdmann assay results into Lennard assay results; this difference is apparently due to differences in hydrolysis times (unpublished data). Mean whole blood 6-TGN concentration of $163 \mathrm{pmol} / 8 \times 10^{8}$ RBCs observed in our study would thus be comparable with a mean RBC 6-TGN concentration of $261 \mathrm{pmol} / 8 \times 10^{8} \mathrm{RBCs}$ using the Lennard assay.

In our study, patients with intermediate TPMT activity had a significantly greater mean RBC 6-TGN concentration compared with patients with normal TPMT activity (253v $\left.151 \mathrm{pmol} / 8 \times 10^{8} \mathrm{RBCs} ; \mathrm{p}=0.002\right)$. However, although an inverse relationship between TPMT activity and RBC 6-TGN concentration was found, patients with intermediate TPMT activity did not exhibit an increased frequency of leucopenia. This lack of leucopenia is not surprising as no patient with AZA or 6-MP treatment duration less than 3.5 months participated (thus no patients with early leucopenia due to decreased TPMT activity participated). As discussed above, clinicians may have elected to use a lower dose of AZA or 6-MP in patients with known intermediate TPMT activity.

Benzoic acid derivatives such as 5-ASA, $\mathrm{N}$-acetyl-5-ASA, sulphasalazine, olsalazine, and balsalazide cause in vitro inhibition of TPMT activity; IC $_{50}$ values for 5-ASA, sulphasalazine, olsalazine, and balsalazide are $1380,104,31$, and $197 \mu \mathrm{M}$, respectively. ${ }^{2021}$ Coadministration of olsalazine and 6-MP in a patient with intermediate TPMT activity led to bone marrow suppression. ${ }^{22}$ We found that patients receiving AZA or 6-MP and mesalamine, sulphasalazine, or olsalazine had a higher mean whole blood 6-TGN concentration and lower mean WBC count than patients receiving AZA or 6-MP alone. Mean plasma concentrations of 5-ASA, $N$-acetyl 5-ASA, sulphasalazine, and olsalazine observed in our study were somewhat below the $\mathrm{IC}_{50}$ values for these compounds. Nevertheless, given the trend towards an increase in whole blood 6-TGN (pharmacokinetic effect) that occurred in our study, and 
the significant decrease in mean WBC (pharmacodynamic effect), it appears that the concentrations of 5-ASA and related compounds found in our study may be sufficient to inhibit TPMT activity in vivo. We have demonstrated elsewhere that addition of mesalamine, sulphasalazine, or balsalazide to stable therapy with AZA or 6-MP in patients with CD results in an increase in whole blood 6-TGN concentrations and a high frequency of leucopenia. ${ }^{10}$

In the largest study to date, we were unable to confirm the usefulness of therapeutic drug monitoring with 6-TGN in patients with IBD treated with AZA or 6-MP. There are methodological differences between our study and previous ones that demonstrated that measuring 6-TGN was useful in this setting. We confirmed the findings of other reports demonstrating an inverse relationship between TPMT activity and whole blood 6-TGN, and the occurrence of a drug interaction between mesalamine, sulphasalazine, or olsalazine and AZA or 6-MP, leading to increased whole blood 6-TGN and a lower mean WBC. With the exception of a single drug interaction study, ${ }^{10}$ all studies conducted to date are observational and do not prospectively test the hypothesis that dose adjustment of AZA or 6-MP to a target whole blood or RBC 6-TGN concentration will improve treatment outcomes in patients with IBD. Our study should serve as a cautionary note that adoption of 6-TGN monitoring into routine clinical practice may be premature at the present time. A randomised, double blind, controlled trial comparing AZA or 6-MP dose optimisation based on 6-TGN concentrations with standard therapy (standard initial dose adjusted only for toxicity) should be conducted. In addition, studies investigating the precise cellular and molecular mechanisms of action of AZA and 6-MP should be undertaken.

Supported by grants from the American College of Gastroenterology, the Mayo Foundation for Research and Education, and grant FD-T-000-886 from the FDA.

1 Pearson DC, May GR, Fick GH, et al. Azathioprine and 6-mercaptopurine in Crohn's disease. A meta-analysis. Ann Intern Med 1995;122:132-42.

2 George J, Present DH, Pou R, et al. The long-term outcome of ulcerative colitis treated with 6-mercaptopurine. Am $\mathcal{F}$ Gastroenterol 1996;91:1711-14.

3 Lennard L. The clinical pharmacology of Lennard L. The clinical pharmacology of
6-mercaptopurine. Eur f Clin Pharmacol 1992;43:329-39.
4 Weinshilboum RM, Sladek SL. Mercaptopurine pharmacogenetics: monogenic inheritance of erythrocyte hiopurine methyltransferase activity. Am f Hum Genet 1980;32:651-62.

5 Lennard L, Van Loon JA, Lilleyman JS, et al. Thiopurine pharmacogenetics in leukemia: correlation of erythrocyte thiopurine methyltransferase activity and 6-thioguanine nucleotide concentrations. Clin Pharmacol Ther 1987;41: 18-25.

6 Bergan S, Bentdal O, Sodal G, et al. Patterns of azathioprine metabolites in neutrophils, lymphocytes, reticulocytes, and erythrocytes: relevance to toxicty and monitoring in recipients of renal allografts. Ther Drug Monit 1997;19:502-9.

7 Lents of renal allografts. Ther Drug Monit 1997;19:502-9. Lennard L, Rees CA, Lilleyman JS, et al. Childhood
leukemia: a relationship between intracellular 6-mercpatopurine metabolites and neutropenia. Br f Clin 6-mercpatopurine metabolte

8 Cuffari C, Theoret Y, Latour S, et al. 6-Mercaptopurine metabolism in Crohn's disease: correlation with efficacy and toxicity. Gut 1996;39:401-6.

9 Dubinsky MC, Lamothe S, Yang HY, et al. Pharmacogenomics and metabolite measurement for Gastroenterology 2000;118:705-13.

10 Lowry PW, Franklin CL, Weaver AL, et al. Leucopenia resulting from a drug interaction between azathioprine or 6-mercaptopurine and mesalamine, sulphasalazine, or bal6-mercaptopurine and mesalam

11 Sandborn WJ, Tremaine WJ, Wolf DG, et al. Lack of effect of intravenous administration on time to respond to azathioprine for steroid-treated Crohn's disease. Gastroenterology 1999;117:527-35.

12 Lilleyman JS, Lennard L. Mercaptopurine metabolism and the risk of relapse in childhood lymphoblastic leukaemia. Lancet 1994;343:1188-90.

13 Irvine EJ, Feagan B, Rochon J, et al. Quality of life: a valid and reliable measure of therapeutic efficacy in the treatment of inflammatory bowel disease. Gastroenterology 1994;106:287-96.

14 Szumlanski CL, Honchel R, Scott MC, et al. Human liver thiopurine methyltransferase pharmacogenetics: biochemical properties, liver-erythrocyte correlation and presence of isozymes. Pharmacogenetics 1992;4:148-59.

15 Erdmann GR, France LA, Bostrom BC, et al. A reversed phase high performance liquid chromatography approach in determining total red blood cell concentrations of 6-thioguanine, 6-mercaptopurine, methylthioguaine, and 6-thioguanine, 6-mercaptopurine, methylthioguaine, and methylmercaptopurine in a patient recei
therapy. Biomed Chromatogr 1990;4:47-51.

16 Awni WM, Braeckman RA, Locke CS, et al. The influence of multiple oral doses of zileuton on the steady-state pharmacokinetics of sulfasalazine and its metabolites, sulfapyridine and $\mathrm{N}$-acetylsulfapyridine. Clin Pharmacokinet 1995; 29(suppl 2):98-104.

17 Yu DK, Elvin AT, Morrill B, et al. Effect of food coadministration on 5-aminosalicylic acid oral suspension bioavailability. Clin Pharmacol Ther 1990;48:26-33.

$18 \mathrm{De}$ Vos M, Verdievel H, Schoonjans R, et al. Highperformance liquid chromatographic assay for the determination of 5-aminosalicylic acid and acetyl-5-aminosalicylic acid concentrations in endoscopic intestinal biopsy in humans. F Chromatogr 1991;564:296-302.

19 Lennard L, Maddocks JL. Assay of 6-thioguanine nucleotide, a major metabolite of azathioprine, 6-mercaptopurine, and 6-thioguanine, in human red blood cells. F Pharm Pharmacol 1983;35:15-18.

20 Szumlanski CL, Weinshilboum RM. Sulphasalazine inhibition of thiopurine methyltransferase: possible mechanism for interaction with 6-mercaptopurine and azathioprine. $\mathrm{Br}$ f Pharmacol 1995;39:456-9.

21 Lowry PW, Szumlanski CL, Weinshilboum RM, et al. Balsalazide and azathioprine or 6-mercaptopurine: evidence for a potentially serious drug interaction. Gastroenterology 1999;116:1505-6.

22 Lewis LD, Benin A, Szumlanski CL, et al. Olsalazine and 6-mercaptopurine-related bone marrow suppression: a possible drug interaction. Clin Pharmacol Ther 1997;62: 464-75. 